\title{
Nanoneurology - drug delivery across the brain protective barriers
}

\begin{abstract}
Neurology is that branch of medicine that deals with the diagnosis and treatment of disorders of the nervous (central, peripheral) system and all effector tissues. This article is concerned with those neurological brain disorders that are difficult to treat because of the brain protective barriers. Such diseases may require the delivery of drugs across the brain protective barriers. This topic is of high interest because of the growing aging population and the consequent relative prevalence of neurological disorders, and brain cancer and inflammation
\end{abstract}

Keywords: Peptide database, Inflammation, Disease, Ventricular surfaces, Tumors
Volume 5 Issue I - 2017

\author{
Alain L Fymat \\ International Institute of Medicine and Science, USAI \\ Correspondence: Alain L Fymat, International Institute of \\ Medicine and Science, California, USA, Tel 760-485-9149; \\ Email alain.fymat@fiimas.org
}

Received: January 24, 2017 | Published: February 09, 2017
Abbreviations:AD, Alzheimer Disease; Apo-E, ApoLipoprotein-E; BBB, Blood Brain Barrier; Brainpeps, BBB Peptide Database; CNS, Central Nervous System; CSF, CerebroSpinal Fluid; EPIC, Engineered Peptide Compound; HAD, HIVAssociated Dementia; HIFU, High Intensity Focused Ultrasound; HIV, HIV Encephalitis; HIV, Human Immunodeficiency Virus; LPR, Lipoprotein Related Protein; MEN, Magneto-Electric Nanoparticles; MS, Multiple Sclerosis; ND, Nano Device; NP, Nano Particle; PD, Parkinson Disease; P-gP, P-Glycoprotein; PLGA, Poly Lactic coGlycolic Acid; PNS, Peripheral Nervous System; RNA, Ribo Nucleic Acid

\section{Diseases}

Alzheimer Disease; Dementia; Encephalitis; Epilepsy; Gliosarcoma; Meningitis; HIV; Multiple Sclerosis; Parkinson Disease

\section{Drugs}

Bradykinin; Casomorphin; Doxoribucin

\section{Introduction}

Neurology (from the Greek: veṽpov-"neuron" and the suffix

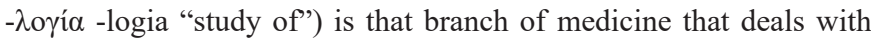
the diagnosis and treatment of disorders of the central nervous system (CNS: brain and spinal cord), the peripheral nervous system (PNS), including the autonomic and somatic subdivisions, their coverings, blood vessels and all effector tissues. In the neurological literature, we find approximately 400 known neural disorders some of which may perhaps be better classified as mental disorders. Some of these may be due to a disruption or failure of the blood brain barrier (BBB) such as, for example: meningitis (an inflammation of the meninges or membranes surrounding the brain and spinal cord; epilepsy (chronic or acute seizures caused by inflammation); multiple sclerosis (MS, a disease of either the immune system or/and the breaking down of the BBB in a section of the brain or spinal cord); Alzheimer disease (AD, a disease in which amyloid beta contained in blood plasma enter the brain and adhere to the surface of astrocytes); possibly prion and prion-like diseases such as Parkinson disease (PD) and AD; HIV encephalitis (HIVE), a precursor of HIV-associated dementia (HAD) in which latent HIV can cross the BBB inside circulating monocytes in the blood stream; and systemic inflammation (sterile or infectious) that may lead to effect on the brain, cause sickness behavior and induce or/and accelerate brain diseases such as MS and PD. There are currently active investigations into treatments for a compromised $\mathrm{BBB}$. As a consequence of the growing aging population, many such neurodegenerative diseases, cancer and infections of the brain will become more prevalent. Of interest here are those disorders requiring treatment by delivery of drugs across the brain protective barriers.

\section{Protective barriers of the brain}

\section{Description}

Being the most delicate organ of the body, the brain is protected against potentially toxic substances by the blood-brain barrier, which restricts the entry of most pharmaceuticals into the brain. The collective term "blood-brain barrier" (BBB) describes four main interfaces between the CNS and the periphery: (a) The BBB proper formed by complex tight junctions between the endothelial cells of the cerebral vasculature. Its primary manifestation is the impermeability of the capillary wall due to the presence of the junctions and a low endocytic activity; (b) the blood-cerebro spinal fluid (CSF) barrier formed by tight junctions between epithelial cells of the choroid plexus. Both (a) and (b) extend down the spinal cord; (c) the outer CSF-brain barrier formed by tight junctions between endothelial cells of the arachnoid vessels (pia arachnoid); and (d) the inner CSF-brain barrier formed by strap junctions between the neuro-ependymal cells lining the ventricular surfaces. It is present only in early development and absent in the adult. ${ }^{1}$ The above barriers and the blood retinal barrier (not studied here) are parts of a whole realm of barriers. The following considerations are limited to the BBB proper.

\section{Function}

The BBB behaves as a continuous lipid bilayer which is the major obstacle preventing the passage of polar and lipid-insoluble substances and drugs that are potentially useful for combating diseases affecting the CNS. In its neuroprotective role, the BBB functions to hinder the delivery of many potentially important diagnostic and therapeutic agents to the brain. Therapeutic molecules and antibodies that might otherwise be effective in diagnosis and therapy do not cross the $\mathrm{BBB}$ in adequate amounts. Essential nutrients are delivered to the brain by selective transport mechanisms (glucose transporter, amino acid transporters, etc.). Although most drugs can enter the brain by passive diffusion through the endothelial cells depending on their lipophilicity, degree of ionization, molecular weight, relative brain 
tissue and plasma bindings, some others can use specific endogenous transporters. In such cases, binding competition on the transporter with endogenous products or nutrients can occur and limits drug transfer. Extensive efforts are therefore being made to come up with drug delivery strategies that would enable the passage of therapeutic molecules safely and effectively. Such strategies involve modifying the drug itself or coupling it to a vector for receptor-mediated or adsorption-mediated transcytosis. ${ }^{2,3}$

\section{Drug targeting difficulties}

The BBB can be a major impediment and the main limitation for the treatment of neurological diseases caused by inflammation, tumors or neurodegenerative disorders the close intercellular contact between cerebral endothelial cells due to tight junctions prevents the passive diffusion of hydrophilic components from the bloodstream into the brain. Thus, many drugs are unable to reach this organ at therapeutic concentrations. Various attempts have been made to overcome the limiting access of drugs to the brain, e.g. chemical modification, development of more hydrophobic analogs or linking an active compound to a specific carrier. Transient opening of the BBB in humans has been achieved by intracarotid infusion of hypertonic mannitol solutions or of bradykinin analogs. Another way to increase or decrease brain delivery of drugs is to modulate the P-glycoprotein (P-gp) whose substrates are actively pumped out of the cell and into the capillary lumen. Many P-gp inhibitors or inducers are available to enhance the therapeutic effects of centrally acting drugs or to decrease central adverse effects of peripherally active drugs. ${ }^{4}$ Nonetheless, overcoming the difficulty of delivering therapeutic agents to specific regions of the brain presents a major challenge to the treatment of most brain disorders.

\section{Mechanisms for drug targeting}

Mechanisms for drug targeting in the brain involve going either "through" or "behind" the BBB:

\section{Modalities for drug delivery/dosage form "through" the BBB}

These entail:

a. Disrupting it by osmotic means. This can be accomplished biochemically by the use of vasoactive substances such as bradykinin or even by localized exposure to high-intensity focused ultrasound (HIFU);

b. Modulating the expression and/or the activity of efflux transporters. Several specific transport systems (via transporters expressed on cerebral endothelial cells) are implicated in the delivery of nutrients, ions and vitamins to the brain; other transporters expressed on cerebral endothelial cells extrude endogenous substances or xenobiotics (which have crossed the cerebral endothelium) out of the brain and into the bloodstream;

c. Using the physiological receptor-dependent BBB transport;

d. Other methods used to get through the BBB by increasing the permeability of the BBB entail the use of endogenous transport systems, including carrier-mediated transporters such as glucose and amino acid carriers; receptor-mediated trancytosis for insulin or transferrin; and the blocking of active efflux transporters such as the transferrin receptor of active efflux transporters such as P-gp. However, vectors targeting BBB transporters, such as the transferrin receptor have been found to remain entrapped in brain endothelial cells of capillaries, instead of being ferried across the $\mathrm{BBB}$ into the cerebral parenchyma; and e. Still, other methods create new viral or chemical vectors to cross the BBB.

\section{Methods for drug delivery "behind" the BBB}

These methods by-pass the BBB by central drug administration. They include intracerebral implantation (such as with needles) and convection-enhanced distribution. Mannitol can be used to bypass the BBB.

\section{Approaches to Enhancing Drug Delivery}

The developmental process for new drugs for the treatment of CNS disorders has not kept pace with progress in molecular neurosciences because most of the new drugs discovered are unable to cross the BBB. The clinical failure of CNS drug delivery may be attributed largely to a lack of appropriate drug delivery systems. Localized and controlled delivery of drugs at their desired site of action is preferred because it reduces toxicity and increases treatment efficiency. The various strategies that have been explored to increase drug delivery into the brain include.

\section{Physiological approaches}

Physiological approaches are utilized to improve the transcytosis capacity of specific receptors expressed across the BBB. Low density lipoproteins related protein (LPR) with engineered peptide compound (EpiC) formed the platform incorporating the Angiopep peptide as a new effective therapeutics.

\section{Chemical delivery systems}

These systems include (a) lipid-mediated transport, (b) the prodrug approach and (c) the lock-in system.

\section{Biological delivery}

In these systems, pharmaceuticals are re-engineered to cross the $\mathrm{BBB}$ via specific endogenous transporters localized within the brain capillary endothelium.

\section{Disruption of the BBB}

An example of BBB disruption is by modification of tight junctions, which causes a controlled and transient increase in the permeability of brain capillaries.

\section{Use of molecular Trojan horses}

Examples are peptidomimetic monoclonal antibodies to transport large molecules, e.g. antibodies, recombinant proteins, nonviral gene medicines or ribonucleic acid (RNA) interference drugs across the BBB.

\section{Particulate drug carrier systems}

For more than 30 years, considerable efforts have been made to enhance the delivery of therapeutic molecules across the vascular barriers of the CNS. The intense search for alternative routes of drug delivery (e.g. intranasal drug delivery, convection-enhanced diffusion and intrathecal/ intraventricular drug delivery systems) has been driven by the need to overcome the physiological barriers of the brain and to achieve high drug concentrations within the brain. The current challenge is to develop drug delivery strategies that will allow the passage of drug molecules through the BBB in a safe and effective manner Receptor-mediated transport systems exist for certain endogenous peptides, such as insulin and transferrin, enabling these molecules to cross the BBB in vivo. The use of polymers for local 
drug delivery has greatly expanded the spectrum of drugs available for the treatment of brain diseases, such as malignant tumours and AD. In addition, various drug delivery systems have been used to enhance drug delivery to the brain, e.g. liposomes, microspheres, nanoparticles, nanogels and bionanocapsules. Recently, microchips and biodegradable polymers have become important in brain tumour therapy.

\section{Nanoparticles}

Nanoparticles are found to be effective careers in delivery of conventional drugs, recombinant proteins, vaccines as well as nucleotides. Nonetheless, more research is needed to determine which strategies will be most effective and how they can be improved for patients with brain tumors. It should be noted that vascular endothelial cells and associated pericytes are often abnormal in tumors and that the BBB may not always be intact in brain tumors. Also, the basement membrane is sometimes incomplete. Other factors, such as astrocytes, may contribute to the resistance of brain tumors to therapy. The potential for using $\mathrm{BBB}$ opening to target specific agents to brain tumors has just begun to be explored.

Nanotechnology may help in the transfer of drugs across the $\mathrm{BBB}$. Indeed, delivering drugs across the BBB is one of the most promising applications of nanotechnology in clinical neuroscience. Nanoparticles (solid colloidal particle ranges from 1 to $1000 \mathrm{~nm}$ in size) could potentially carry out multiple tasks in a predefined sequence. Nanoparticles (NP) are solid colloidal particles ranging in size from 1 to $1000 \mathrm{~nm}$ that are utilized as drug delivery agents. The use of NPs to deliver drugs to the brain across the BBB may provide a significant advantage to current strategies. The following nanotechnologies are available:

\section{Liposomes}

Recently, researchers have been trying to build liposomes loaded with nanoparticles to gain access through the BBB.

\section{Peptides}

Peptides are able to cross the BBB through various mechanisms, opening new diagnostic and therapeutic avenues. However, the BBB transport data are scattered across the literature over different disciplines, using different methodologies and reporting different influx or efflux aspects. Therefore, a comprehensive BBB peptide database (Brainpeps) was constructed to collect the data available in the literature. Brainpeps currently contains BBB transport information with positive as well as negative results. The database is a useful tool to prioritize peptide choices for evaluating different BBB responses or studying quantitative structure-property and relationships of peptides. Because a multitude of methods have been used to assess the BBB behaviour of compounds, the methods and their responses were classified. For example, casomorphin is a heptapeptide able to pass through the BBB. Moreover, the relationships between the different BBB transport methods have been clarified and visualized.

\section{Radiolabeled polyethylene glycol coated hexadecylcyanoacrylate nanospheres}

A significant amount of research in this area has been spent exploring methods of nanoparticle-mediated delivery of antineoplastic drugs to tumors in the CNS. For example, radiolabeled polyethylene glycol coated hexadecylcyanoacrylate nanospheres targeted and accumulated in a rat gliosarcoma. However, this method is not yet ready for clinical trials, due to the accumulation of the nanospheres in surrounding healthy tissue.

\section{Polyalkylcyanoacrylate or poly-lactic-co-glycolic acid (PLGA) nanoparticles with polysorbate 80 or poloxamer 188}

Another, more promising approach, is the coating of polyalkylcyanoacrylate or poly-lactic-co-glycolic acid (PLGA) nanoparticles with polysorbate 80 or poloxamer 188 . Due to this coating, the particles adsorb apolipoproteins E (apo-E) or A-1 from the blood and thus interact with the LRP or with the scavenger receptor followed by transcytosis across the BBB into the brain. These particles loaded with doxorubicin for the treatment of glioblastomas are presently in a phase I clinical trial.

\section{Magneto-electric nanoparticles}

Recently, a novel class of multifunctional nanoparticles known as magneto-electric nanoparticles (MENs) has been discovered for externally controlled targeted delivery and release of drug(s) across the BBB as well as wireless stimulation of cells deep in the brain. This approach depends more on the field control and less on the cellular microenvironment. In vitro and in vivo (on mice) experiments to prove the feasibility of using MENs to release a drug across the BBB on demand and wirelessly stimulate the brain have been conducted by the research group of Prof. Sakhrat Khizroev at Florida International University.

\section{Nano devices}

Because of their size and functionalization characteristics, NPs are able to penetrate and facilitate drug delivery through the BBB. There are a number of mechanisms and strategies found to be involved in this process, which is based on the type of nanomaterials used and its combination with therapeutic agents. As just discussed, such materials include liposomes, polymeric nanoparticles and non-viral vectors of nano-sizes for CNS gene therapy, etc. The primary advantage of NP carrier technology is that NPs mask the BBB-limiting characteristics of the therapeutic drug molecule. Furthermore, this system may slow drug release in the brain, decreasing peripheral toxicity. Influencing manufacturing factors (type of polymers and surfactants, NP size, and the drug molecule) related to movement of the drug delivery agent across the BBB. Currently, reports evaluating NPs for brain delivery have studied anesthetic and chemotherapeutic agents. However, physiological factors such as phagocytic activity of the reticuloendothelial system and protein opsonization may limit the amount of brain delivered drug. ${ }^{7,8}$ Various strategies are used based on different types of nanomaterial and combinations with therapeutic agents.

At present NPs are found to play a significant advantage over the other methods of available drug delivery systems for delivery of therapeutics to the CNS. Although the use of nanotechnology is expected to reduce the need for invasive procedures for delivery of therapeutics to the CNS, some devices such as implanted catheters and reservoirs will still be needed. Nanomaterials can improve the safety and efficacy of such devices. Nano-engineered probes can deliver drugs at the cellular level using nanofluidic channels. There is some concern about the safety of nanoparticle entry in the brain and these needs to be resolved before human use. Although there is presently no approved nanotechnology-based CNS drug as yet that incorporates nano biotechnology, the future for such developments is promising. ${ }^{9}$

Nanomaterials improve the safety and efficacy level of drug delivery devices in brain targeting and nano engineered devices can deliver the drugs at cellular levels through nano-fluidic channels. 
Whereas the different drug delivery systems described previously such as liposomes, microspheres, nanoparticles, nanogels and nano bio capsules have been used to improve the bioavailability of the drug in the brain, microchips and biodegradable polymeric nano particulate carriers may be more effective therapeutically in treating brain tumor. Nano particulate drug delivery systems improve the pharmacokinetic strategies of the drug molecules such as: biodistribution; bioavailability; and drug release characteristics in a controlled and effective manner with site specific drug delivery targeting to tissue or cell; and reduction in toxic manifestation. ${ }^{10}$

Notwithstanding the several advantages of nanoparticles and nanodevices, some devices such as implanted catheters and reservoirs will still be needed to overcome the problems in effective drug delivery to the CNS.

\section{Acknowledgments}

None.

\section{Conflicts of interest}

None.

\section{References}

1. Stolp HB, Liddelow SA, Sá-Pereira I et al. Immune Responses at Brain Barriers and Implications for Brain Development and Neurological Function in Later Life. Front Integr Neurosci. 2013;7:61.
2. Temsamani J, Rousselle C, Reese AR et al. Vector-mediated drug delivery to the brain. Expert Opin Biol Ther. 2001;1(5):773-782.

3. Scherrmann JM Drug delivery to brain via the blood-brain barrier. Vascul Pharmacol. 2002;38(6):349-354.

4. Jolliet Riant P, Tillemont JP Drug transfer across the blood-brain barrier and improvement of brain delivery. Fundam Clin Pharmacol. 1999;13(1):16-26.

5. Weiss N, Miller F, Cazaubon S et al. Blood-brain barrier part III: therapeutic approaches to cross the blood-brain barrier and target the brain. Rev Neurol (Paris). 2010;166(3):284-288.

6. Patel MM, Goyal BR, Bhadada S et al. Getting into the brain: Approaches to enhance brain drug delivery. CNS Drugs. 2009;23(1):35-58.

7. Lockman PR, Mumper RJ, Khan MA et al. Nanoparticle Technology for Drug Delivery across the Blood-Brain Barrier. Drug Dev Ind Pharm. 2002;28(1):1-13.

8. Chen Y, Dalwadi G, Benson HA Drug delivery across the blood-brain barrier. Curr Drug Deliv. 2004;1(4):361-376.

9. Jain KK Nano biotechnology-Based Drug Delivery to the Central Nervous System. Neurodegener Dis. 2007;4(4):287-291.

10. Dinda SC, Pattnaik G Nano biotechnology-Based Drug Delivery in Brain Targeting. Curr Pharm Biotechnol. 2013;14(15):1264-1274. 